\title{
Novel Targeted Therapy for Precursor B-Cell Acute Lymphoblastic Leukemia: Anti-CD22 Antibody-MXD3 Antisense Oligonucleotide Conjugate
}

\author{
Noriko Satake, ${ }^{1,2}$ Connie Duong, 1,2 Sakiko Yoshida, ${ }^{1,2}$ Michael Oestergaard, ${ }^{3}$ Cathy Chen, ${ }^{1,2}$ \\ Rachael Peralta, ${ }^{3}$ Shuling Guo, ${ }^{3}$ Punit P Seth, ${ }^{3}$ Yueju Li, ${ }^{4}$ Laurel Beckett, ${ }^{4}$ Jong Chung, ${ }^{1}$ Jan Nolta, ${ }^{2}$ \\ Nitin Nitin, ${ }^{5}$ and Joseph M Tuscano ${ }^{6}$ \\ ${ }^{1}$ Department of Pediatrics, University of California, Davis, United States of America; ${ }^{2}$ Stem Cell Program, University of California, \\ Davis, United States of America; ${ }^{3}$ Ionis Pharmaceuticals, Carlsbad, California, United States of America; ${ }^{4}$ Department of Public \\ Health Sciences, University of California, Davis, United States of America; ${ }^{5}$ Departments of Food Science \& Technology and \\ Biological \& Agricultural Engineering, University of California, Davis, United States of America; and ${ }^{6}$ Department of Internal \\ Medicine, University of California, Davis, United States of America
}

\begin{abstract}
The exponential rise in molecular and genomic data has generated a vast array of therapeutic targets. Oligonucleotide-based technologies to down regulate these molecular targets have promising therapeutic efficacy. However, there is relatively limited success in translating this into effective in vivo cancer therapeutics. The primary challenge is the lack of effective cancer cell-targeted delivery methods, particularly for a systemic disease such as leukemia. We developed a novel leukemiatargeting compound composed of a monoclonal antibody directly conjugated to an antisense oligonucleotide (ASO). Our compound uses an ASO that specifically targets the transcription factor MYC-associated factor $X$ (MAX) dimerization protein 3 (MXD3), which was previously identified to be critical for precursor B-cell (preB) acute lymphoblastic leukemia (ALL) cell survival. The MXD3 ASO was conjugated to an anti-cluster of differentiation-22 (CD22) antibody ( $\alpha$ CD22 Ab) that specifically targets most preB ALL. We demonstrated that the $\alpha$ CD22 Ab-ASO conjugate treatment showed MXD3 protein knockdown and leukemia cell apoptosis in vitro. We also demonstrated that the conjugate treatment showed cytotoxicity in normal B cells, but not in other hematopoietic cells, including hematopoietic stem cells. Furthermore, the conjugate treatment at the lowest dose tested $(0.2 \mathrm{mg} / \mathrm{kg}$ Ab for 6 doses - twice a week for $3 \mathrm{wks})$ more than doubled the mouse survival time in both Reh (median survival time 20.5 versus $42.5 d, p<0.001$ ) and primary preB ALL (median survival time 29.3 versus $63 d, p<0.001$ ) xenograft models. Our conjugate that uses $\alpha$ CD22 Ab to target the novel molecule MXD3, which is highly expressed in preB ALL cells, appears to be a promising novel therapeutic approach.
\end{abstract}

Online address: http://www.molmed.org

doi: $10.2119 /$ molmed.2015.00210

\section{INTRODUCTION}

Precursor B-cell (preB) acute lymphoblastic leukemia (ALL) is the most common type of ALL $(1,2)$. The prognosis for adult preB ALL is poor, with overall cure rates of approximately $40 \%$ (3-5). Although the overall cure rate of pediatric preB ALL has improved dramatically through the introduction of intensive combination chemotherapy since the 1960s, the prognosis for certain subtypes remains very poor, with cure rates of approximately $30 \%(6-8)$. In addition, current chemo and radiation therapies can cause late effects, including

Address correspondence to Noriko Satake, Section of Hematology/Oncology, Department of Pediatrics, 2516 Stockton Blvd., Sacramento, CA 95817, USA. Tel: +1-916-734-2781; Fax: 17-916-456-2236; E-mail: nsatake@ucdavis.edu. Submitted September 22, 2015; Accepted for Publication July 13, 2016; Published Online (wWw.molmed.org) July 22, 2016.

IIV

Feinstein Institute for Medical Research Northwell Health"

secondary malignancies $(9,10)$. Targeted therapies for ALL have the potential to be more effective and have fewer side effects than current treatments.

Antibody (Ab)-based therapeutics are promising targeted treatment strategies that are currently being investigated for ALL $(11,12)$. Although monoclonal antibodies (mAbs), as a single agent, have limited therapeutic efficacy, they have improved efficacy when combined with standard induction therapy (13). Furthermore, mAbs have been shown to have a role as cell-targeting agents as in Ab-drug (14-16) or -immunotoxin (17-20) conjugates. More recently, there have been promising results with $\mathrm{Ab}$ constructs that redirect 
T cells, such as bispecific T-cell engager (BiTE) Abs $(21,22)$ and chimeric antigen receptor (CAR)-based T-cell therapies (23-25). Antisense oligonucleotides (ASOs) have enormous potential as gene-targeted agents that have high specificity (26-30). Over the past decade, clinical trials using ASO therapies have demonstrated modest efficacy for cancers, including chronic lymphocytic leukemia (31), prostate and lung cancers (32-35). Major challenges with ASO-based cancer therapies remain, however, and include non-specific delivery and inefficient intracellular uptake (36-38). Conjugates of $\mathrm{mAb}$ and ASO can deliver ASOs to target leukemia cells for selective knockdown of leukemia-specific genes in vivo, minimizing non-specific ASO delivery. To date only a few Ab-ASO conjugates have been reported $(39,40)$.

$\mathrm{CD} 22$ is a B-lineage restricted surface molecule that modulates B-cell receptor signaling (41). It is an ideal target for Abbased therapy against B-cell malignancies because of its high expression and rapid internalization upon $\mathrm{Ab}$ binding $(17,42,43)$. MAX dimerization protein 3 (MXD3) is a basic-helix-loop-helix-leucine-zipper transcription factor that is part of the v-myc avian myelocytomatosis viral oncogene homolog (MYC)/MAX/MXD transcriptional network involved in cellular proliferation (44-46). Previously, we demonstrated that MXD3 functions as an anti-apoptotic protein and that knockdown of MXD3 can be a novel effective therapeutic strategy for preB ALL in vitro $(47,48)$. In this study, we developed a novel leukemia-targeting compound using MXD3 ASO conjugated to anti-CD22 Ab ( $\alpha$ CD22 Ab) for preB ALL. We demonstrated that the $\alpha \mathrm{CD} 22$ Ab-MXD3 ASO conjugate has significant in vitro and in vivo therapeutic efficacy using preclinical xenograft mouse models of human preB ALL.

\section{MATERIALS AND METHODS}

\section{ASO and $\mathrm{Ab}$}

ASOs were designed and synthesized using standard solid phase oligonucleotide synthetic methods (Ionis Pharmaceuticals). The MXD3 ASO sequence is 5'-CACAG GGACG CATAA C-3'. It is a 3-10-3 (S)-cEt gapmer, wherein the three nucleosides at the $5^{\prime}$-end and the three nucleosides at the $3^{\prime}$-end comprise 2', 4'-constrained-2'-O-Ethyl Bridged Nucleic acid (cEt), and the ten middle nucleosides are 2'-deoxynucleosides (49). The negative control ASO sequence, which has no known homology to mammalian genes and has minimal non-specific effects, is $5^{\prime}$-CCTTC CCTGA AGGTT CCTCC-3'. It is a 5-10-5 2'-methoxyethyl (MOE) gapmer, wherein the five nucleosides at the $5^{\prime}$-end and the five nucleosides at the 3 '-end comprise MOE modifications, and the ten middle nucleosides are 2'-deoxynucleosides. All internucleoside linkages are phosphorothioate linkages. The cytosine bases are 5-methylcytosines. The 5'-end of each oligonucleotide was modified to comprise a cyclooctyne for subsequent click chemistry conjugation to an azide-labeled antibody via 1,3-dipolar cycloaddition (50). The 5'-DBCO-TEG phosphoramidite (Glen Research) was coupled to the 5'-end of each oligonucleotide using standard solid phase methods to form a phosphodiester linkage between the oligonucleotide and the 5'-DBCO-TEG moiety. Ammonia deprotection was completed at room temperature for a minimum of $48 \mathrm{~h}$.

The $\alpha \mathrm{CD} 22$ mAbs ( $\alpha$ CD22 Ab: JT22.1) were generated by the fusion of NS-1 myeloma cells with spleen cells from BALB/c mice immunized with baby hamster kidney cells transfected with human CD22 cDNA encoding the transmembrane domain (BP 2208-2263) and extracytoplasmic domains 1 and 2 (BP 57-867). Hybridomas were screened and selected based on the ability of the mAbs to specifically bind to $293 \mathrm{~T}$ cells that were transfected with CD22 extracytoplasmic domains 1 and 2, but not with non-transfected control cells. Positive clones were subcloned twice. The $\alpha \mathrm{CD} 22$ Abs were purified using protein G Hi-Trap columns (Amersham). JT22.1 was assessed for CD22 ligand blocking as previously described (51). The isotype of the JT22.1 was determined to be IgG1 using a Mouse mAb Isotyping kit (Amersham).

\section{Cell Lines and Patient-Derived Leukemia Cells}

The human preB ALL cell line Reh was purchased from ATCC and the Jurkat cell line was obtained from Dr. Kit Lam's Lab at UC Davis. Cells were maintained at $37^{\circ} \mathrm{C}$ in a $5 \% \mathrm{CO}_{2}$ incubator in RPMI 1640 (Life Technologies) supplemented with $10 \%$ heat-inactivated fetal bovine serum, $100 \mathrm{U} / \mathrm{mL}$ penicillin and $100 \mu \mathrm{g} / \mathrm{mL}$ streptomycin (Thermo Scientific), 0.25\% D-glucose (SigmaAldrich), $1 \mathrm{mmol} / \mathrm{L}$ sodium pyruvate and $10 \mathrm{mmol} / \mathrm{L}$ HEPES buffer (Thermo Scientific).

Primary leukemia samples were collected from patients with informed consent based on our institutionally approved IRB protocol. Normal blood cells were collected with IRB approval from anonymized discarded apheresis bags from healthy donors. B cells (lymphocytes) and CD34 + hematopoietic stem cells (HSCs) were isolated using magnetic beads (Miltenyi Biotec) and non-B cells were collected from the counterpart of B-cell isolation. Cell purity of the isolated B cells and CD34 + HSCs was confirmed by flow cytometry (FC500 Beckman Coulter) using anti-CD19 and anti-CD34 $\mathrm{Ab}$, respectively (BD Biosciences).

\section{$\alpha$ CD22 Ab-ASO Conjugate}

The method for Ab-ASO conjugation is summarized in Supplementary Figure S1. $\alpha$ CD22 Ab was incubated with NHS-PEG4-Azide (azide) (Thermo Scientific) (dissolved in dimethyl sulfoxide (DMSO)) in phosphate buffered saline (PBS) at a molar ratio of 1:100 for $3 \mathrm{~h}$ at $4^{\circ} \mathrm{C}$. Excess azide was removed using a Zeba 7 k MW desalting column (Thermo Scientific). The azide-conjugated $\mathrm{Ab}$ was then incubated with cyclooctyne-modified ASO in water at a $1: 20$ ratio at $37^{\circ} \mathrm{C}$ for $30 \mathrm{~min}$ to form the $\alpha \mathrm{CD} 22 \mathrm{Ab}$-ASO conjugates. Excess ASO was removed as described above. The above reaction method was optimized by titrating the molar ratio of $\alpha \mathrm{CD} 22 \mathrm{Ab}$, azide and $\mathrm{ASO}$, and the incubation time of $\alpha \mathrm{CD} 22$ $\mathrm{Ab}$-azide with ASO. The conjugation of 
the $\mathrm{Ab}$ and $\mathrm{ASO}$ was validated using SDS-PAGE gel assays. For the experiments in which free $\alpha \mathrm{CD} 22 \mathrm{Ab}$ or ASO were used as a control, the equivalent amount of $\alpha \mathrm{CD} 22 \mathrm{Ab}$ in the conjugate, or ASO in the conjugation reaction, was used.

\section{In Vitro Treatment with $\alpha$ CD22 Ab-ASO Conjugate}

Reh cells were plated at 20,000 cells in $125 \mu \mathrm{L}$ medium per well in 48-well plates in triplicate for each treatment group and time point. The cells were treated with $\alpha \mathrm{CD} 22 \mathrm{Ab}-\mathrm{ASO}$ conjugates (MXD3 or control) at indicated concentrations, or left untreated. MXD3 protein expression and live cell counts were assessed at 4 , $8,24,48$ or $72 \mathrm{~h}$ after treatment. The cells were incubated with indicated treatment for $4 \mathrm{~h}$ in complete growth medium, which was then replaced with fresh growth medium.

\section{Immunocytochemistry and Fluorescent Image Intensity Quantification}

Cells were fixed with $10 \%$ buffered formalin and stained with anti-MXD3 monoclonal mouse $\mathrm{Ab}$ (Neuromab) and secondary goat anti-mouse Ab-Alexa488 (Life Technologies) as previously described (47). Nuclei were stained with switch locations of data: 40,6-diamidino-2-phenylindole (DAPI) (Life Technologies). The MXD3 protein expression levels were quantified by mean fluorescent intensity (MFI) using Image J (NIH) as previously described $(47,52)$.

\section{Immunoblotting}

Frozen whole cell pellets (1 million cells per treatment group) were denatured and boiled with NuPAGE Sample Reducing agent (Thermo Fisher Scientific) in PBS at $100^{\circ} \mathrm{C}$ for $10 \mathrm{~min}$ before being run on a 4 to 20\% Tris-Glycine sodium dodecyl sulfate polyacrylamide gel electrophoresis (SDSPAGE) gel. Protein was transferred to a nitrocellulose membrane using wet transfer system at $100 \mathrm{~V}$ for $1 \mathrm{~h}$. Blots were blocked with 5\% non-fat dry milk in Tris-buffered saline (TBS) with 1\% tween-20 (TBST) for
$1 \mathrm{~h}$ at room temperature, then incubated with primary antibodies in 5\% non-fat dry milk in TBST overnight at $4^{\circ} \mathrm{C}$. After the overnight incubation, blots were washed with TBST 3 times for 10 min each before incubation with secondary antibodies in $5 \%$ non-fat dry milk in TBST for $1 \mathrm{~h}$ at room temperature. Blots were washed 2 times with TBST and once with TBS before incubation with SuperSignal West Pico Chemiluminescent kit according to the manufacturer's instructions (Thermo Scientific). Primary antibodies and incubation conditions were 1:1,000 for mouse anti-MXD3 (Neuromab) and 1:2,000 for rabbit anti-histone (Abcam). Secondary antibodies and incubation conditions were 1:1,000 for goat anti-mouse horseradish peroxidase (HRP) antibody and 1:2,000 for goat anti-rabbit HRP antibody.

\section{Apoptosis Assay}

Cell apoptosis was measured by annexin V (BD Biosciences) or caspase activity using the Caspase 3/7 Glo kit (Promega) as previously described (47). Annexin $\mathrm{V}$ was measured by flow cytometry using the FC500. Caspase level was measured by a Centro LB 960 Microplate Luminometer (Berthold Technologies). Both experiments were performed twice.

\section{In Vivo Therapeutic Studies in Mice}

All procedures were performed in compliance with our institutionally approved animal care protocol in the barrier facility vivarium at the Institute for Regenerative Cures in accordance with AALAC. Human leukemia xenograft models were established with either Reh or patient-derived leukemia samples from two different donors and 5- to 10-wk-old female NOD/SCID/ IL2Rg-/- (NSG) mice. The two primary samples were from patients who were diagnosed as high-risk based on the current diagnostic criteria (53). For the patient-derived leukemia models, passage 3 of serially transplanted NSG mice were used. Five million leukemia cells were transplanted via intravenous (Reh) or intra-tibial (patient-derived cells) injection. Treatment was initiated $24 \mathrm{~h}$ after leukemia inoculation, with intravenous injection of PBS, unconjugated $\mathrm{Ab}$ and $\mathrm{ASO}$ or conjugate at indicated doses. Mice were randomly assigned to each treatment group. Mice were treated twice a week for $3 \mathrm{wks}$, and each cohort included 4, 6 or 8 mice. In one of the patient-derived models, complete blood count (CBC) and chemistry panels were checked from one representative mouse per cohort weekly for $4 \mathrm{wks}$. Mice were monitored daily until they were moribund, or, for the Reh model, developed hind limb paralysis. Mice were sacrificed and leukemia cells were harvested from bone marrow (BM). Human leukemia cells were confirmed by flow cytometry using anti-HLA-ABC Ab (BioLegend) and B-cell leukemia panels, including anti-human CD10, 19, 20 and 22 Abs (BD Biosciences).

For treatment effect assessment, Reh leukemia engrafted mice were used with a high dose of $\alpha \mathrm{CD} 22 \mathrm{Ab}-\mathrm{MXD} 3 \mathrm{ASO}$ conjugate $(10 \mathrm{mg} / \mathrm{kg}$ of the $\mathrm{Ab})$. Reh engraftment was confirmed by Giemsa staining and phenotyping on tibial BM aspirates on d 17 after leukemia inoculation. To assess in vivo targeted effects a dose of either PBS or $\alpha$ CD22 Ab-MXD3 ASO conjugate was given on d 18, with 2 mice per cohort. Eight and $24 \mathrm{~h}$ after treatment, mice were euthanized and harvested BM was tested for live cell counts, MXD3 knockdown, and apoptosis.

\section{Statistical Analysis}

In vitro analyses had five main goals: to assess the effect of conjugated and unconjugated antibody on MXD3; to characterize dose response; to assess therapeutic efficacy via cytotoxicity; to correlate cytotoxicity with MXD3 knockdown; and to assess specificity. Results of in vitro studies were summarized descriptively by mean and standard error (SE) and linear regression was used to compare outcomes for conjugate to comparison groups. Analysis of variance (ANOVA) was also performed at each time point followed by pairwise multiple comparisons (Tukey studentized range), with cell counts log-transformed 
where necessary to meet assumptions. For in vivo studies to assess therapeutic efficacy, previous work in our laboratory with the human leukemia xenograft model showed survival times for control group animals that were brief (approximately $3 \mathrm{wks}$ ) and very consistent (SD less than $5 d$ ) (54). Thus sample sizes of 4 to 8 animals per group were predicted by power analysis to be adequate to detect clinically important shifts in survival times, for example an increase to $5 \mathrm{wks}$, with very high power (>90\% even after adjustment for multiple comparisons). Survival was summarized by Kaplan-Meier plots and compared by log-rank tests and linear regression analyses with 95\% confidence intervals (CI). No samples or animals were excluded for analysis. The analyses were carried out in Prism version 6, SAS version 9.3, or R. Command-line data are available on request.

All supplementary materials are available online at www.molmed.org.

\section{RESULTS}

$\alpha C D 22$ Ab-MXD3 ASO Conjugate Shows MXD3 Knockdown in a DoseDependent Manner in Reh Cells

Our group and others have shown that nearly $100 \%$ of Reh cells express CD22 whereas Jurkat cells do not $(47,54)$. We have previously shown that MXD3 is highly expressed in malignant B cells, including Reh cells, but is very low in Jurkat cells (47). Therefore, Reh cells were used to assess the conjugate, with Jurkat cells serving as a negative control. Reh cells treated with various concentrations of the conjugate were assessed for MXD3 expression by immunocytochemistry which showed a dose-dependent MXD3 protein knockdown at 4 and $24 \mathrm{~h}$ after treatment (Supplementary Figure S2A). Protein levels were quantified using MFI $(52,55,56)$. Using an average of both time points, the percentage knockdown was $13 \%(0.0005 \mu \mathrm{mol} / \mathrm{L}), 34 \%(0.005 \mu \mathrm{mol} / \mathrm{L})$, $47 \%(0.05 \mu \mathrm{mol} / \mathrm{L})$ and $75 \%(0.5 \mu \mathrm{mol} / \mathrm{L})$ (Supplementary Figure S2B). The MXD3 protein levels were also confirmed by immunoblotting (Supplementary Figure S3). To confirm that the MXD3 knockdown was mediated by the conjugate, and not the $\alpha \mathrm{CD} 22 \mathrm{Ab}$, Reh cells were treated with the conjugate and/or naked $\alpha \mathrm{CD} 22 \mathrm{Ab}$. The MXD3 protein level was modestly affected by treatment with naked $\alpha \mathrm{CD} 22 \mathrm{Ab}$ at concentrations of 0.5 (18\% knockdown) and $5 \mu \mathrm{mol} / \mathrm{L}(27 \%$ knockdown) (Supplementary Figures S4A, S4C). Furthermore, MXD3 knockdown by the conjugate treatment was partially inhibited when unconjugated $\alpha \mathrm{CD} 22$ $\mathrm{Ab}$ was added simultaneously at concentrations equal or 10 times higher than the conjugated $\mathrm{Ab}$ (Supplementary Figures S4B, S4C). When treating with $0.5 \mu \mathrm{mol} / \mathrm{L}$ of the conjugate, co-culture with a 1- or 10-fold concentration of the unconjugated (naked) $\mathrm{Ab}$ resulted in a MXD3 knockdown from $86 \%$ to $73 \%$ or $53 \%$, respectively. For the $0.05 \mu \mathrm{mol} / \mathrm{L}$ conjugate, the knockdown was reduced from $74 \%$ to $75 \%$ or $63 \%$ (1- and 10 -fold naked $\mathrm{Ab}$ added, respectively).

\section{$\alpha C D 22$ Ab-MXD3 ASO Conjugate Treatment Induces Apoptosis in Reh Cells}

We chose the highest concentration of the conjugate $(0.5 \mu \mathrm{mol} / \mathrm{L}$ of the $\mathrm{Ab})$ for the subsequent in vitro studies to ensure demonstration of therapeutic efficacy. To confirm the specificity of the MXD3 ASO, a non-specific ASO conjugate ( $\alpha \mathrm{CD} 22$ Ab-control ASO conjugate) was created and used as a control. Reh cells treated with the $\alpha \mathrm{CD} 22 \mathrm{Ab}-\mathrm{MXD} 3 \mathrm{ASO}$ conjugate showed a $62 \%$ and $55 \%$ reduction in MXD3 protein expression compared with untreated $(* * p=0.008)$ or $\alpha \mathrm{CD} 22$ Ab-control ASO treated cells $\left({ }^{*} p=0.032\right)$, respectively, $4 \mathrm{~h}$ after treatment (Figures 1A, B). We next assessed cell viability after treatment using annexin $\mathrm{V}$ (Figures 1C, D). Untreated or $\alpha \mathrm{CD} 22$ Ab-control ASO conjugate-treated cells showed few annexin $\mathrm{V}$ positive cells (average $2.8 \%$ and $9 \%$ at $2 \mathrm{~h}$, and $2.9 \%$ and $9.3 \%$ at $4 \mathrm{~h}$, respectively) whereas the $\alpha \mathrm{CD} 22$ Ab-MXD3 ASO conjugate-treated cells showed significantly higher levels of annexin $\mathrm{V}$ positive cells (average
$28 \%$ at $2 \mathrm{~h}$, and $51 \%$ at $4 \mathrm{~h}$ ) (Figure 1D, ANOVA $\left.{ }^{* * *} p<0.001\right)$. Caspase levels also showed a significant increase in the $\alpha \mathrm{CD} 22$ Ab-MXD3 ASO conjugate-treated cells compared with controls at both 2 and $4 \mathrm{~h}$ after treatment (Figure 1E, ANOVA $\left.{ }^{* * *} p<0.001\right)$.

\section{$\alpha C D 22$ Ab-MXD3 ASO Conjugate Affects Normal B Cells, But Not Hematopoietic Stem Cells}

Human B cells express low levels of MXD3 whereas CD34 + HSCs and non-B cells have negligible levels of MXD3 (47). We investigated potential cytotoxic effects of the conjugate on B cells as well as on CD34 + HSCs and non-B cells. Isolated B cells and CD34 + HSCs had purity greater than 95\% (data not shown). These cells were treated with the $\alpha$ CD22 Ab-MXD3 ASO, an equimolar amount of free $\alpha \mathrm{CD} 22 \mathrm{Ab}$ plus free MXD3 ASO, or left untreated. To demonstrate leukemiaspecific delivery and therapeutic efficacy of the Ab-ASO conjugate, but not each component (either ASO or Ab) separately, we used free $\mathrm{Ab}$ plus free MXD3 ASO as a control. B cells treated with the $\alpha \mathrm{CD} 22 \mathrm{Ab}-\mathrm{MXD} 3 \mathrm{ASO}$ conjugate or free Ab plus MXD3 ASO showed a 74\% and $24 \%$ reduction in MXD3 protein expression $4 \mathrm{~h}$ after treatment compared with untreated, respectively (Figures 2A, B, ANOVA $p=0.062)$. CD34 + HSCs and non-B cells treated with identical conditions showed no significant difference in MXD3 protein expression between all treatments (data not shown). B cells treated with the $\alpha \mathrm{CD} 22 \mathrm{Ab}-\mathrm{MXD} 3 \mathrm{ASO}$ conjugate showed accelerated cell death in vitro compared with minimal cytotoxicity in both CD34 + HSCs and non-B cells (Figure 2C; ANOVA ${ }^{* * *} p<0.001$ for B cells, $p=0.96$ for non-B cells and $p=0.75$ for CD34 + HSCs).

\section{$\alpha$ CD22 Ab-MXD3 ASO Conjugate Shows Significant Therapeutic Efficacy in Reh Xenograft Mouse Models}

We tested the in vivo therapeutic efficacy of the conjugate in a xenograft mouse model using the Reh cell line. All the mice that received PBS died of 


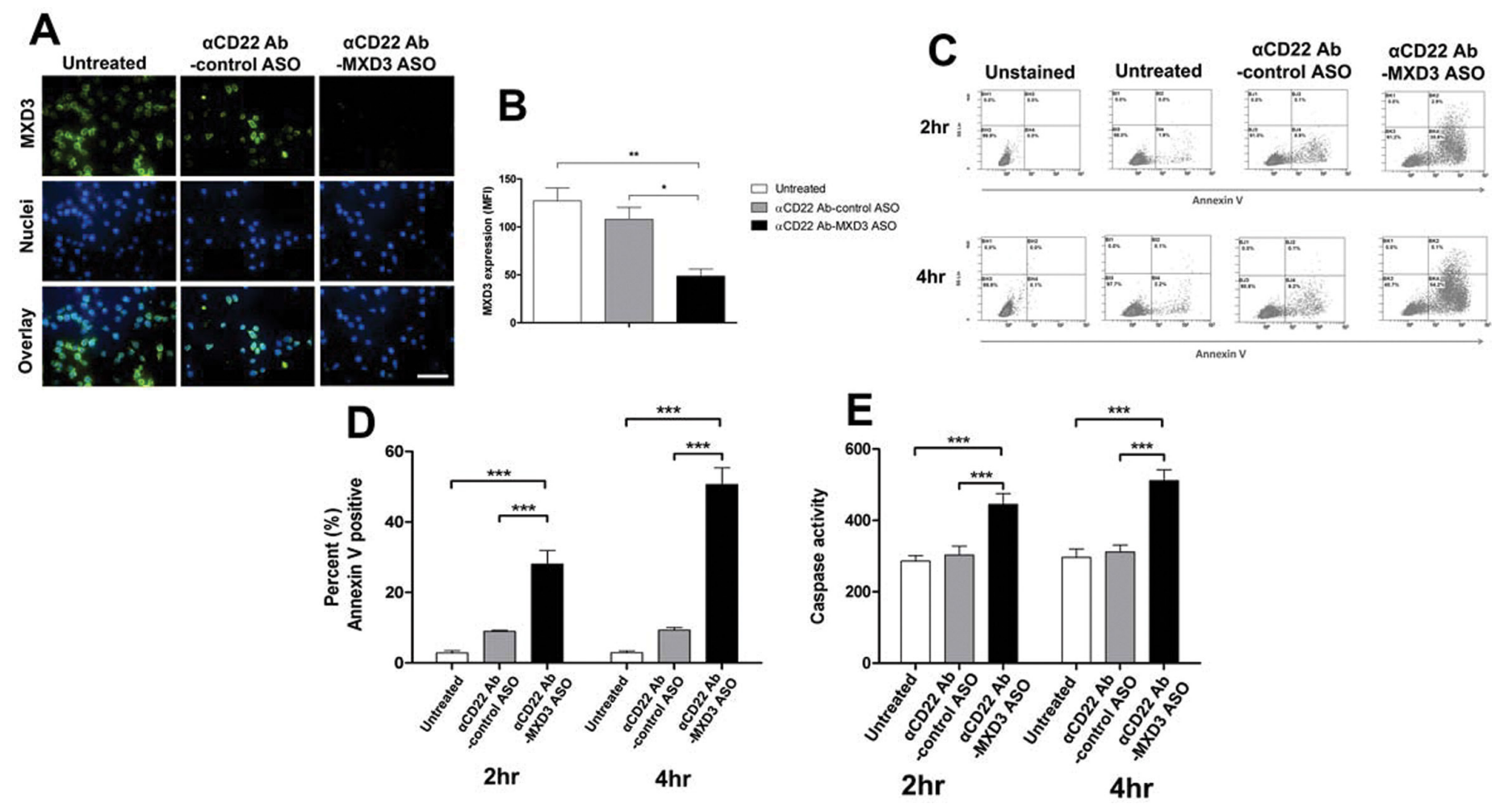

Figure 1. $\alpha$ CD22 Ab-MXD3 ASO conjugate demonstrates MXD3 knockdown leading to cell apoptosis in preB ALL cells. (A) Reh cells treated with $\alpha$ CD22 Ab-MXD3 or $\alpha$ CD22 Ab-control ASO conjugate, or left untreated, were measured for MXD3 protein expression by fluorescent immunocytochemistry $4 \mathrm{~h}$ after treatment. The concentration of the conjugate in each well was $0.5 \mathrm{umol} / \mathrm{L}$. Images were acquired at 40x magnification/1.4 numerical aperture at room temperature using a Nikon Ti-U inverted microscope and NIS-Elements BR software. Scale bar indicates $50 \mu \mathrm{m}$. The overlay pictures show a composite image of both MXD3 protein (with Alexa488) and nuclei (with DAPI). The images shown are from one representative experiment out of three experiments. (B) Mean fluorescence intensity (MFI) was used to quantify MXD3 protein expression. Each bar represents the average MFI of all measured cells per treatment type from three independent experiments. MXD3 $\alpha$ CD22 Ab conjugate versus untreated $\left({ }^{* *} p=0.008\right)$ versus $\alpha C D 22$ Ab-control ASO conjugate $\left({ }^{*} p=0.032\right)$. (C) Cell apoptosis measured by annexin $V$ using flow cytometry. Dot plots shown are from one representative experiment out of three experiments. (D) Quantification of annexin $\vee$ positive cells. Data are means from three independent experiments. Error bars represent SEM ( $n=3$ for each time point). The cells treated with $\alpha$ CD22 Ab-MXD3 ASO conjugate showed significantly more annexin $V$ positive cells than either the untreated cells $\left({ }^{* * *} p<0.001\right)$ or those treated with the $\alpha \mathrm{CD} 22$ Ab-control ASO conjugate $\left({ }^{* * *} p<0.001\right)$, at both 2 and 4 h. (E) Cell apoptosis measured by caspase 3 and 7. Cells were treated the same way as in (D) with the same control. Histograms represent caspase measured by luminescence signal at 2 and $4 \mathrm{~h}$ after treatment. Data are an average of three independent experiments in triplicate. Error bars represent SEM ( $n=9$ for each time point). Cells treated with the $\alpha$ CD22 Ab-MXD3 ASO conjugate showed significantly higher average caspase than either the untreated cells $\left({ }^{* * *} p<0.001\right)$ or those treated with the $\alpha C D 22$ Ab-control ASO conjugate $\left({ }^{* * *} p<0.001\right)$, at both 2 and $4 \mathrm{~h}$.

leukemia on d 21 and 22 as expected (Figure 3). All the mice that received free $\alpha C D 22 \mathrm{Ab}$ plus free MXD3 ASO died of leukemia between d 19 and 55 (median survival time was d 21, 36, 53 for the 1, 5, $10 \mathrm{mg} / \mathrm{kg}$ free $\mathrm{Ab}$ dose, respectively), with no difference in survival between the $1 \mathrm{mg} / \mathrm{kg}$ Ab dose and PBS, but an increase in survival of $15.5 \mathrm{~d}$ for each increase in dose (95\% CI 8.9-22.1 d, $\left.{ }^{* * *} p<0.001\right)$. The mice that received the conjugate, regardless of the dose, survived significantly longer (median survival time was d 42.5, 62, 110 and 112.5 for the $0.2,1,5$ and $10 \mathrm{mg} / \mathrm{kg} \mathrm{Ab}$ dose, respectively) than the mice that received PBS or equivalent unconjugated controls. The increase in survival was 50.1 $\mathrm{d}$ at the $1 \mathrm{mg} / \mathrm{kg}$ Ab dose compared with the unconjugated control (95\% CI 39.6$60.6 \mathrm{~d},{ }^{* * *} p<0.001$ ) with a dose response effect of $25 \mathrm{~d}$ for each dose increase or decrease $(* * *<0.001)$. At the time of harvest, all the mice were confirmed to have developed human leukemia (human leukocyte antigen (HLA) and CD22 positive) (Supplementary Table S1A). During treatment, the mice in all the treatment groups remained healthy without weight loss (Supplementary Figure S5A).

To confirm that the in vivo therapeutic efficacy of the conjugate is mediated by MXD3 knockdown leading to leukemia 


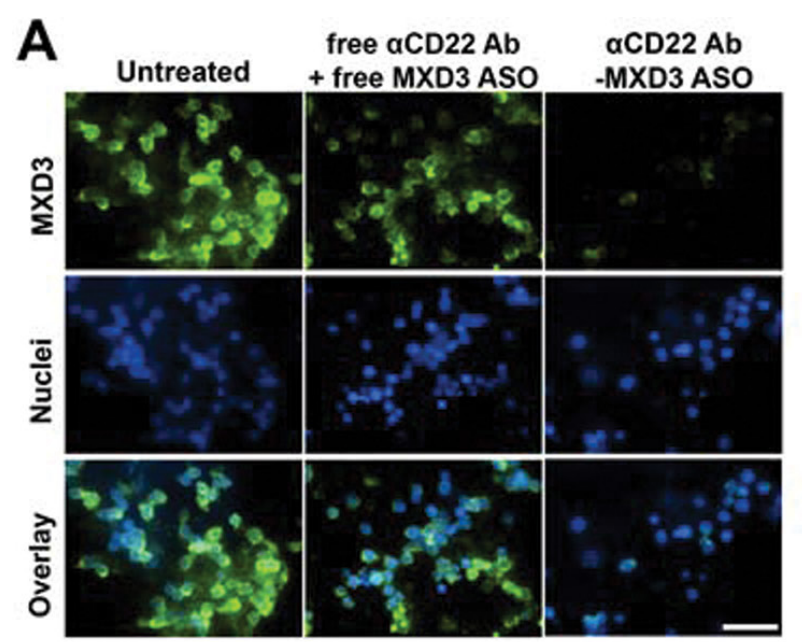

B

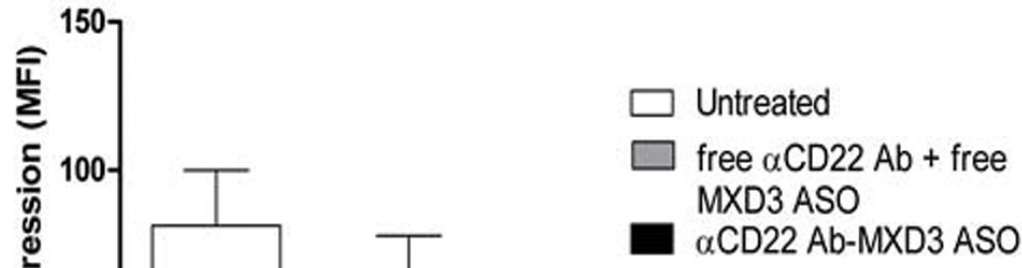

C

B-cells
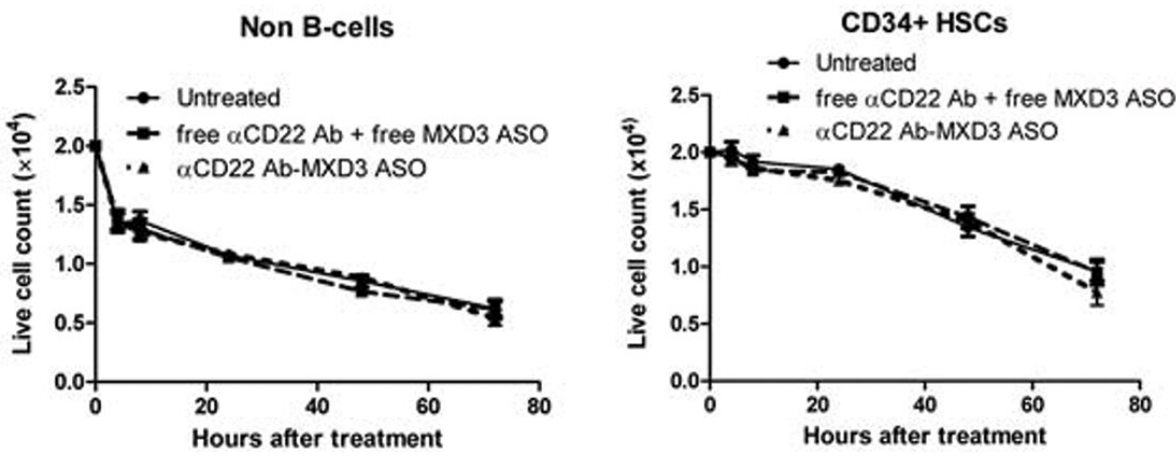

Figure 2. aCD22 Ab-MXD3 ASO conjugate has cytotoxicity in normal B cells, but not in HSCs. (A) Normal B cells express low levels of MXD3 and treatment with the $\alpha$ CD22 Ab-MXD3 ASO conjugate showed knockdown. Images were acquired at 40x magnification/1.4 numerical aperture at room temperature using a Nikon Ti-U inverted microscope and NIS-Elements BR software. Scale bar indicates $50 \mu \mathrm{m}$. Free $\alpha \mathrm{CD} 22$ $\mathrm{Ab}$ (azide-conjugated) + free MXD3 ASO also showed low levels of non-specific knockdown. The images shown are from one representative experiment out of three experiments. (B) MXD3 protein knockdown quantified using MFI. Each bar represents the average MFI of all measured cells per treatment type from three independent experiments. (C) Accelerated cell death in B cells treated with the $\alpha$ CD22 Ab-MXD3 ASO conjugate. Data points indicate mean values of independent cell counts in triplicate from three independent experiments in triplicate. Data as mean \pm SEM $(n=9)$. The MXD3 $\alpha C D 22$ Ab-ASO conjugate versus free $\alpha C D 22$ Ab (azide-conjugated) + free MXD3 ASO (*** $p<0.001$ ).

cell apoptosis, we performed a pilot study treating Reh engrafted mice with high tumor burden ( 3 wks after leukemia inoculation) with a single dose Ab-ASO conjugate treatment. The Reh cells harvested from the BM of the conjugate-treated mice showed lower levels of MXD3 expression than PBStreated mice both at 8 (Figure $4 \mathrm{~A}$ ) and $24 \mathrm{~h}$ (data not shown) after treatment. The BM cells of the conjugate-treated mice showed a much higher percentage of apoptotic Reh cells which were double positive for CD22 and annexin $\mathrm{V}: 33.2 \%$ versus $4.8 \%$ at $8 \mathrm{~h}$ and $29.5 \%$ versus $2.5 \%$ at $24 \mathrm{~h}$ (Figure $4 \mathrm{~B}$ ). These results demonstrate that the in vivo therapeutic efficacy of the $\alpha \mathrm{CD} 22$ Ab-MXD3 ASO conjugate was mediated by MXD3 knockdown-induced apoptosis in leukemic cells.

\section{$\alpha C D 22$ Ab-MXD3 ASO Conjugate Shows Significant Therapeutic Efficacies in Two Preclinical preB ALL Human Patient-Derived Xenograft Mouse Models}

We next tested the in vivo therapeutic efficacy of the conjugate in two different patient-derived leukemia xenograft models. A summary of known clinical, phenotypic, and molecular characteristics are included in Supplementary Table S2. We have previously shown that primary preB ALL cells, including the two samples used in this study, express CD22 and MXD3 $(47,54)$. The conjugate, even at the two lowest doses tested in the Reh model, showed significant therapeutic efficacy in both models. In the sample A model, all the mice that received PBS or free $\alpha \mathrm{CD} 22 \mathrm{Ab}$ plus free MXD3 ASO died of leukemia between $\mathrm{d} 27$ and 32 (median survival time 29 and $29.5 \mathrm{~d}$, respectively), whereas 


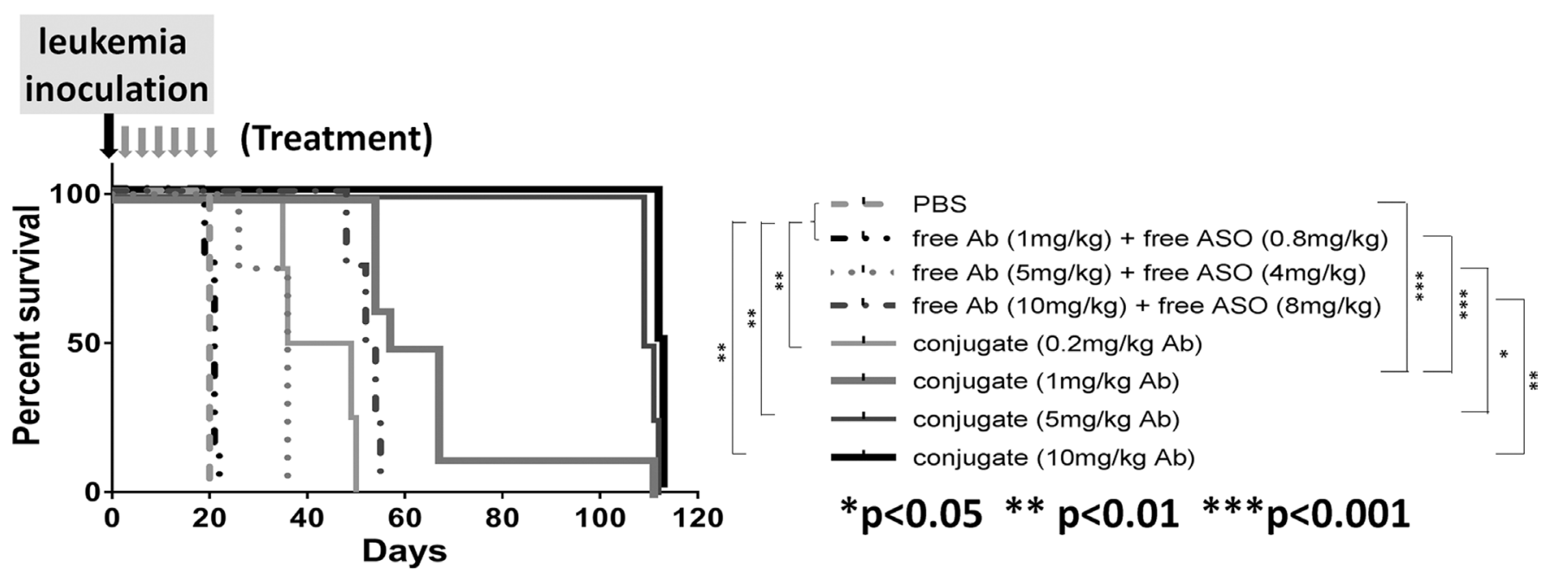

Figure 3. aCD22 Ab-MXD3 ASO conjugate shows significant in vivo dose-dependent therapeutic efficacy in Reh human leukemia mouse model. Kaplan-Meier survival curve for mice inoculated with Reh cells and treated with the $\alpha$ CD22 Ab-MXD3 ASO conjugate. Data from two independent experiments were combined ( $n=4$ or 8 ). In the first experiment, the mice were treated with PBS, free aCD22 Ab (1 mg/kg) plus free MXD3 ASO (0.8 mg/kg), and two different doses of the $\alpha$ CD22 Ab-MXD3 ASO conjugate (0.2 mg/kg or $1 \mathrm{mg} / \mathrm{kg}$ of the $\mathrm{Ab}$ ). In the second experiment, the mice were treated with free $\alpha \mathrm{CD} 22 \mathrm{Ab}(1,5 \mathrm{or} 10 \mathrm{mg} / \mathrm{kg}) \mathrm{plus}$ free $\mathrm{MXD} 3 \mathrm{ASO}$ $(0.8,4$ or $8 \mathrm{mg} / \mathrm{kg})$, respectively, and three different doses of the $\alpha$ CD22 Ab-MXD3 ASO conjugate (1, 5 or $10 \mathrm{mg} / \mathrm{kg}$ of the Ab). PBS versus conjugate at any dose $(0.2,1,5$ or $10 \mathrm{mg} / \mathrm{kg}$ of the $\mathrm{Ab})\left({ }^{* *} p<0.01,{ }^{* * *} p<0.001,{ }^{* *} p<0.01\right.$ or ${ }^{* *} p<0.01$, respectively). Free $\alpha \mathrm{CD} 22$ $\mathrm{Ab}(1 \mathrm{mg} / \mathrm{kg})$ plus free MXD3 ASO $(0.8 \mathrm{mg} / \mathrm{kg})$ versus conjugate at any dose $\left(0.2,1,5\right.$ or $10 \mathrm{mg} / \mathrm{kg}$ of the Ab) $\left({ }^{* *} p<0.01,{ }^{* * *} p<0.001\right.$, ${ }^{* *} p<0.01$ or ${ }^{* *} p<0.01$, respectively). Free Ab plus free ASO versus conjugate at the equivalent dose of the Ab (1,5 or $\left.10 \mathrm{mg} / \mathrm{kg}\right)$ $\left({ }^{* * *} p<0.001,{ }^{*} p=0.01\right.$ or ${ }^{* *} p<0.01$, respectively).

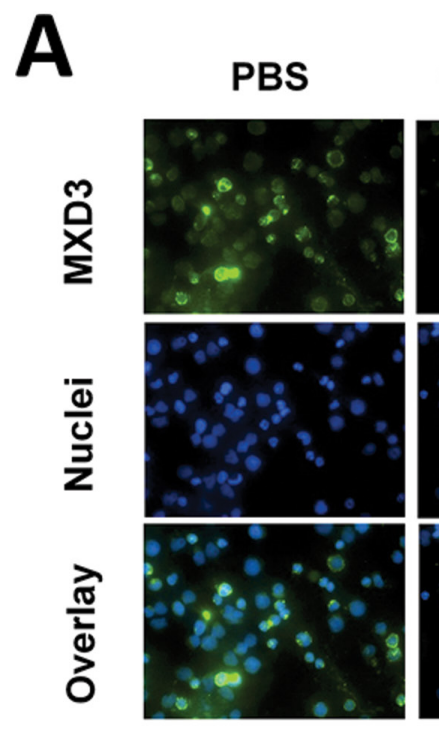

Conjugate

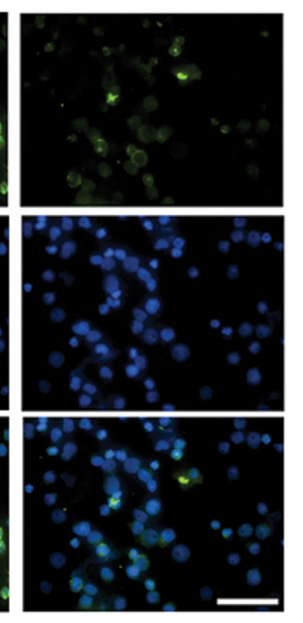

B
CD22

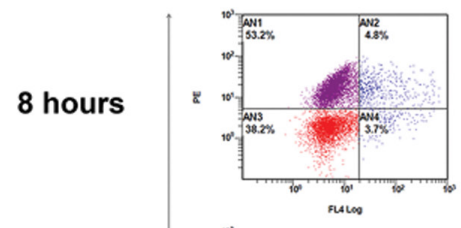

24 hours

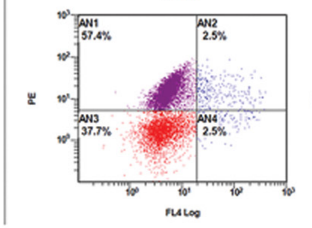

\section{Conjugate}
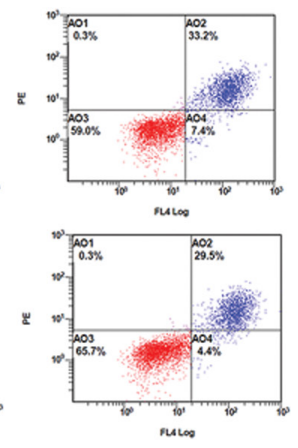

\section{Annexin V}

Figure 4. $\alpha \mathrm{CD} 22$ Ab-MXD3 ASO conjugate shows in vivo anti-leukemic effects against engrafted leukemia cells in mice. (A) The $\alpha C D 22$ Ab-MXD3 ASO conjugate treatment showed MXD3 knockdown in the engrafted Reh cells at $8 \mathrm{~h}$ after treatment. MXD3 protein expression was measured in the cells harvested from BM. Images were acquired at 40x magnification/1.4 numerical aperture at room temperature using a Nikon Ti-U inverted microscope and NIS-Elements BR software. Scale bar indicates $50 \mu \mathrm{m}$. (B) The $\alpha \mathrm{CD} 22$ Ab-MXD3 ASO conjugate treatment induced cell apoptosis in the engrafted Reh cells at 8 and $24 \mathrm{~h}$ after treatment. Cells were stained for human CD22 and annexin V. Red dots (lower quadrants): murine cells (CD22 negative), purple dots (upper left quadrant): live Reh cells (CD22 positive annexin $\vee$ negative), and blue dots (upper right quadrant): apoptotic Reh cells (CD22 and annexin $\vee$ positive) in BM. 


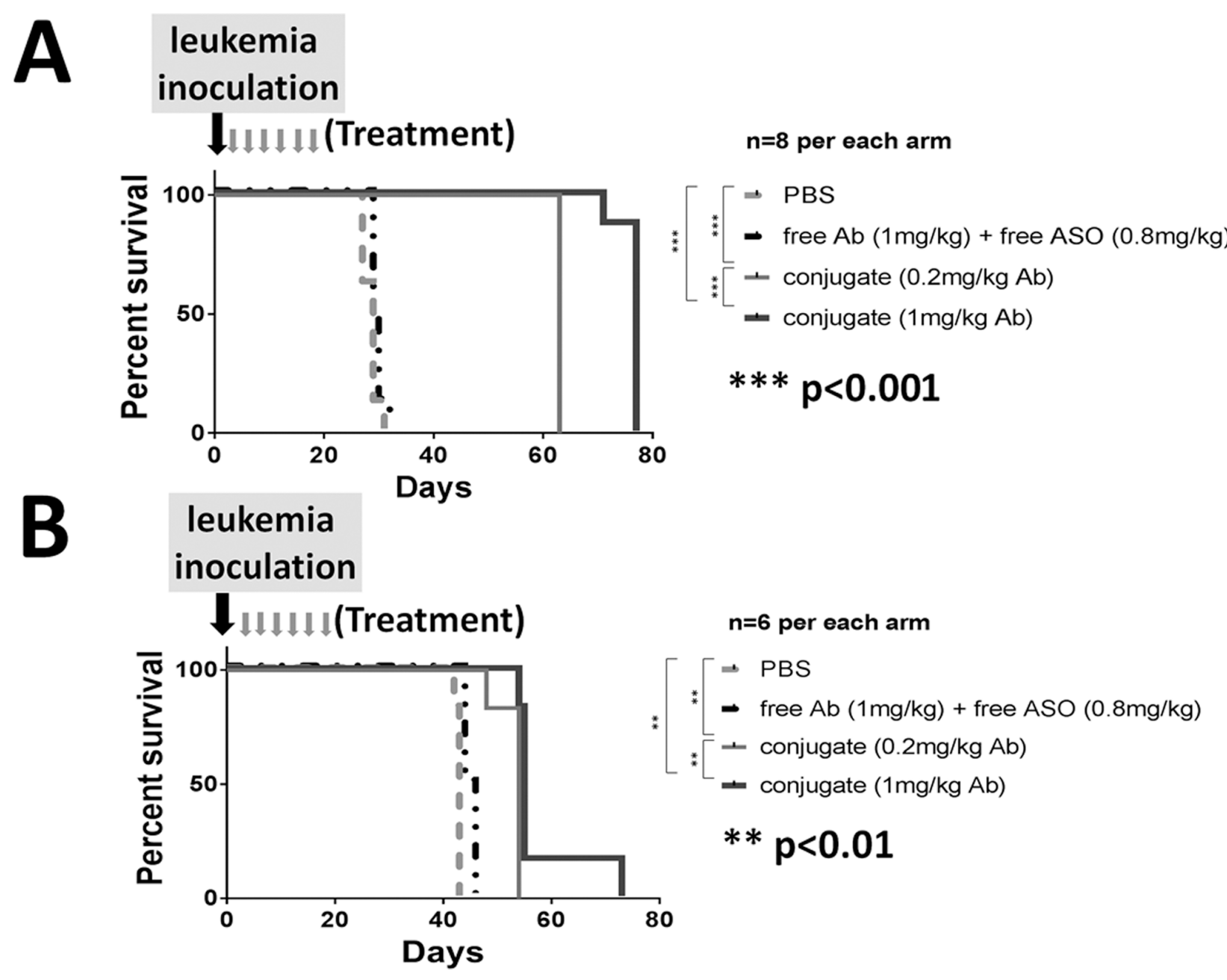

Figure 5. $\alpha$ CD22 Ab-MXD3 ASO conjugate shows significant in vivo therapeutic efficacy in two human patient-derived leukemia mouse models. Kaplan-Meier survival curves for mice inoculated with two patient-derived leukemia cells (A and B). The mice were treated with PBS, free $\alpha$ CD22 $\mathrm{Ab}(1 \mathrm{mg} / \mathrm{kg})$ plus free MXD3 ASO $(0.8 \mathrm{mg} / \mathrm{kg})$, and two different doses of the $\alpha$ CD22 Ab-MXD3 ASO conjugate $(0.2 \mathrm{mg} / \mathrm{kg}$ or $1 \mathrm{mg} / \mathrm{kg}$ of the Ab). (A) PBS versus conjugate at both doses (***p<0.001). Conjugate $0.2 \mathrm{mg} / \mathrm{kg}$ versus $1 \mathrm{mg} / \mathrm{kg}$ (of the Ab) $\left({ }^{* * *} p<0.001\right.$ ). ( $n=8$ ). (B) PBS versus conjugate at both doses $\left({ }^{* *} p<0.01\right.$ ). Conjugate $0.2 \mathrm{mg} / \mathrm{kg}$ versus $1 \mathrm{mg} / \mathrm{kg}$ (of the Ab) $\left.{ }^{* *} p<0.01\right) .(n=6)$

those that received the conjugate, at 0.2 or $1 \mathrm{mg} / \mathrm{kg} \mathrm{Ab}$ dose, survived significantly longer (median survival time 63 and $77 \mathrm{~d}$, respectively). Median improvement in survival time for 0.2 or $1 \mathrm{mg} / \mathrm{kg}$ Ab dose was $33.8 \mathrm{~d}$ (range 31 to $36 \mathrm{~d},{ }^{* * *} p<0.001$ ) and $47.8 \mathrm{~d}$ (range 45 to $\left.50 \mathrm{~d},{ }^{* * *} p<0.001\right)$, respectively (Figure 5A). In the sample B model, all the mice that received PBS or free Ab plus free ASO died of leukemia between $\mathrm{d} 42$ and 46 (median survival time 43 and $45 \mathrm{~d}$, respectively), whereas those that received the conjugate, at either dose, survived significantly longer (median survival time 53 and $57 \mathrm{~d}$, respectively). Median improvement for 0.2 or $1 \mathrm{mg} / \mathrm{kg} \mathrm{Ab}$ dose was $10 \mathrm{~d}$ (range 8 to $12 \mathrm{~d},{ }^{* *} p<0.01$ ) and $11 \mathrm{~d}$ (range 9 to $13 \mathrm{~d},{ }^{* *} p<0.01$ ), respectively (Figure 5B). At the time of harvest, all the mice were confirmed to have developed human leukemia (HLA and CD22 positive) (Supplementary Tables S1B, S1C). Toxicity was assessed in the sample A model during the treatment, which showed essentially no hematologic or non-hematologic toxicities (Supplementary Table S3 and Supplementary Figure S5B).

\section{DISCUSSION}

Given the abundance of genetic information now available, a multitude of highly specific therapeutic targets are available. The use of oligonucleotides, such as ASO and small interfering RNA (siRNA), has been studied as an emerging method to target specific RNA sequences $(27,57,58)$. However, clinical translation of these techniques has had limited therapeutic success in oncology (31-33). The primary challenge is target specific and efficient intracellular delivery of oligonucleotides $(26,27,36-38)$. To overcome this limitation, we have 
developed an effective ASO-conjugated $\mathrm{Ab}$ delivery method for the treatment of preB ALL.

We demonstrated leukemia-specific Ab-mediated ASO delivery that resulted in target knockdown, using $\alpha \mathrm{CD} 22 \mathrm{Ab}$ as the vehicle for delivery of MXD3 ASOs, which was confirmed at the protein level. Although the mechanism of intracellular trafficking of the conjugated ASOs is unknown, our study provides proof of concept that $\alpha \mathrm{CD} 22 \mathrm{Ab}$ effectively mediates leukemia cell-specific, intracellular delivery of intact ASO molecules both in vitro (Figure 1) and in vivo (Figures 3-5). In this study, MXD3 protein knockdown was confirmed as early as $4 \mathrm{~h}$ after ASO was added to the cells (Figure 1 and Supplementary Figures S2, S3). We also observed the same early protein knockdown (measured at $8 \mathrm{~h}$ after a single conjugate injection) in our in vivo study (Figure 4A). The mechanism of action of the $\alpha \mathrm{CD} 22-\mathrm{ASO}$ conjugate remains unclear since the rapid kinetics of MXD3 protein knockdown is not consistent with inhibition of MXD3 mRNA levels (data not shown). Interestingly, the same phenomenon (early MXD3 protein knockdown without confirmation of mRNA knockdown) was observed with several different MXD3 ASO sequences (data not shown), as well as MXD3 siRNA $(47,48)$, with or without $\alpha \mathrm{CD} 22 \mathrm{Ab}$ as a vehicle. These data suggest that the ASO conjugate may mediate MXD3 translational inhibition and thus would explain the inability to detect a reduction in MXD3 transcript levels $(59,60)$. MXD3 is a transcription factor, with a short half-life of only up to $30 \mathrm{~min}$ in Reh cells (unpublished data). Further studies are necessary to assess the specific mechanism of MXD3 knockdown in leukemia cells.

$\alpha \mathrm{CD} 22 \mathrm{Ab}$ has been used as a therapeutic (61-63) or therapeutic vehicle $(17,19,63,64)$; however, our study is the first to demonstrate its use as a vehicle for delivery of ASO for preB ALL. Free MXD3 ASOs used in this study demonstrated some in vitro therapeutic efficacy (data not shown). Our in vivo study also suggests that free ASOs may be more effective at higher doses (Figure 3). However, this would allow non-specific delivery, increasing the potential for off-target effects. Our data support the hypothesis that the $\alpha \mathrm{CD} 22 \mathrm{Ab}$ is the key to effective leukemia cell-specific intracellular ASO delivery. While naked $\alpha$ CD22 $\mathrm{Ab}$ can be therapeutic as a single agent, it is very encouraging that the dose of the $\alpha \mathrm{CD} 22 \mathrm{Ab}$ used in our in vivo studies was only about $1 / 20$ of the previously described single agent (Ab alone) therapeutic dose $(62,65)$.

It is highly promising that our conjugate demonstrated consistent and striking in vivo therapeutic efficacy in a cell line-derived and in two high-risk patient-derived preB ALL xenograft models (Figures 3, 5). While there were slight differences in the CD22 surface expression and MXD3 expression levels in the two patient samples, the conjugate showed efficacy in both samples. Studies of $\alpha$ CD22 Ab-targeted therapy in CD22-positive lymphoma showed no correlation between efficacy and the level of target (CD22) expression $(66,67)$. It is necessary to understand these results to develop a customized (personalized) dose/regimen for individual patients.

Assessment of off-target effects in murine models of human cancer is often limited by inter-species target heterogeneity; however, it is important to note that the MXD3 ASO used in this study targets the same sequence of human and murine MXD3 mRNA, allowing us to study off-target effects to some extent. It is promising that the treatment, including free MXD3 ASO, did not show any significant toxicities (Supplementary Figure S5 and Supplementary Table S3). Our in vitro studies, as expected, showed that the conjugate affected normal human B cells, but not CD34 + HSCs (Figure 2). Further studies, including in vivo studies using mice engrafted with human hematopoietic cells, are warranted to investigate potential off-target and side effects and optimize the dose and schedule of the conjugate. Although some B-cell toxicity seems to be unavoidable, toxicity has been shown to be tolerable in other B-cell-targeting agents, such as Rituximab $(13,68)$, CARbased T-cell therapies (23-25) and BiTE antibody $(21,22)$.

\section{CONCLUSION}

The results of the present study demonstrate the therapeutic efficacy and safety profile of a novel $\alpha \mathrm{CD} 22$ Ab-MXD3 ASO conjugate in preclinical models of human preB ALL. Given the high degree of phenotyptic and molecular specificity, and significant efficacy with minimal toxicity, this conjugate has the potential for multiple applications in conjunction with current drugs, or other targeted therapeutics, to create more effective and less toxic therapies for preB ALL. This work represents an important advance in technology that allows effective in vivo ASO delivery that has the potential to be modified for use in many cancers and other diseases.

\section{ACKNOWLEDGMENTS}

The authors thank Betty Ratliff for assisting with primary sample collections.

\section{DISCLOSURE}

MO, SG, PPS and RP are employees and stockholders of Ionis Pharmaceuticals. This work was supported by research funding from The Hartwell Foundation, Keaton Raphael Memorial Foundation, National Center for Advancing Translational Sciences, NIH, through grant \#UL1 TR000002, and CTSC-MCRTP (Satake) and the California Institute for Regenerative Medicine and NIH Transformative grant R01GM099688 (Nolta). Statistical support was provided through the Biostatistics Shared Resource, UC Davis Comprehensive Cancer Center Support Grant P30CA093373.

\section{REFERENCES}

1. Pui CH, Mullighan CG, Evans WE, Relling MV. (2012) Pediatric acute lymphoblastic leukemia: where are we going and how do we get there? Blood. 120:1165-74.

2. Schrappe M, et al. (2000) Long-term results of four consecutive trials in childhood ALL performed by the ALL-BFM study group from 1981 to 1995. Berlin-Frankfurt-Munster. Leukemia. 14:2205-22. 
3. Hunault M, et al. (2004) Better outcome of adult acute lymphoblastic leukemia after early genoidentical allogeneic bone marrow transplantation (BMT) than after late high-dose therapy and autologous BMT: a GOELAMS trial. Blood. 104:3028-37.

4. Pui CH, Evans WE. (2006) Treatment of acute lymphoblastic leukemia. N. Engl. J. Med. 354:166-78.

5. Faderl S, et al. (2010) Adult acute lymphoblastic leukemia: concepts and strategies. Cancer. 116:1165-76.

6. Pui CH. (1995) Childhood leukemias. N. Engl. J. Med. 332:1618-30.

7. Pui CH, Robison LL, Look AT. (2008) Acute lymphoblastic leukaemia. Lancet. 371:1030-43.

8. Pui CH, Evans WE. (2013) A 50-year journey to cure childhood acute lymphoblastic leukemia. Semin. Hematol. 50:185-96.

9. Schmiegelow K, et al. (2013) Second malignant neoplasms after treatment of childhood acute lymphoblastic leukemia. J. Clin. Oncol. 31:2469-76.

10. Mody R, et al. (2008) Twenty-five-year follow-up among survivors of childhood acute lymphoblastic leukemia: a report from the Childhood Cancer Survivor Study. Blood. 111:5515-23.

11. Bhojwani D, Pui CH. (2013) Relapsed childhood acute lymphoblastic leukaemia. Lancet Oncol. 14: e205-17.

12. Daver N, O'Brien S. (2013) Novel therapeutic strategies in adult acute lymphoblastic leukemia- $\mathrm{a}$ focus on emerging monoclonal antibodies. Curr. Hematol. Malig. Rep. 8:123-31.

13. Thomas DA, et al. (2006) Chemoimmunotherapy with hyper-CVAD plus rituximab for the treatment of adult Burkitt and Burkitt-type lymphoma or acute lymphoblastic leukemia. Cancer. 106:1569-80.

14. Sievers EL, Linenberger M. (2001) Mylotarg: antibody-targeted chemotherapy comes of age. Curr. Opin. Oncol. 13:522-7.

15. Senter PD, Sievers EL. (2012) The discovery and development of brentuximab vedotin for use in relapsed Hodgkin lymphoma and systemic anaplastic large cell lymphoma. Nat. Biotechnol. 30:631-7.

16. Lewis Phillips GD, et al. (2008) Targeting HER2-positive breast cancer with trastuzumab-DM1, an antibody-cytotoxic drug conjugate. Cancer Res. 68:9280-90.

17. Wayne AS, et al. (2010) Anti-CD22 immunotoxin RFB4(dsFv)-PE38 (BL22) for CD22-positive hematologic malignancies of childhood: preclinical studies and phase I clinical trial. Clin. Cancer Res. 16:1894-903.

18. Wayne AS, FitzGerald DJ, Kreitman RJ, Pastan I. (2014) Immunotoxins for leukemia. Blood. 123:2470-7.

19. Kantarjian $H$, et al. (2012) Inotuzumab ozogamicin, an anti-CD22-calecheamicin conjugate, for refractory and relapsed acute lymphocytic leukaemia: a phase 2 study. Lancet Oncol. 13:403-11.

20. Younes A, et al. (2012) Phase I multidoseescalation study of the anti-CD19 maytansinoid immunoconjugate SAR3419 administered by intravenous infusion every 3 weeks to patients with relapsed/refractory B-cell lymphoma. J. Clin. Oncol. 30:2776-82.

21. Topp MS, et al. (2011) Targeted therapy with the T-cell-engaging antibody blinatumomab of chemotherapy-refractory minimal residual disease in B-lineage acute lymphoblastic leukemia patients results in high response rate and prolonged leukemia-free survival. J. Clin. Oncol. 29:2493-8.

22. Topp MS, et al. (2014) Phase II Trial of the Anti-CD19 Bispecific T cell-Engager Blinatumomab Shows Hematologic and Molecular Remissions in Patients With Relapsed or Refractory B-Precursor Acute Lymphoblastic Leukemia. J. Clin. Oncol. 32:4134-40.

23. Kochenderfer JN, Rosenberg SA. (2013) Treating B-cell cancer with T cells expressing anti-CD19 chimeric antigen receptors. Nature reviews. Clinical oncology 10:267-76.

24. Kenderian SS, Ruella M, Gill S, Kalos M. (2014) Chimeric antigen receptor T-cell therapy to target hematologic malignancies. Cancer Res. 74:6383-9.

25. Maus MV, Grupp SA, Porter DL, June CH. (2014) Antibody-modified T cells: CARs take the front seat for hematologic malignancies. Blood. 123:2625-35.

26. Manoharan M. (2002) Oligonucleotide conjugates as potential antisense drugs with improved uptake, biodistribution, targeted delivery, and mechanism of action. Antisense Nucleic Acid Drug Dev. 12:103-28.

27. Juliano R, Alam MR, Dixit V, Kang H. (2008) Mechanisms and strategies for effective delivery of antisense and siRNA oligonucleotides. Nucleic Acids Res. 36:4158-71.

28. Kurreck J. (2003) Antisense technologies. Improvement through novel chemical modifications. Eur. J. Biochem. 270:1628-44.

29. Crooke ST. (2004) Progress in antisense technology. Annu. Rev. Med. 55:61-95.

30. Bennett CF, Swayze EE. (2010) RNA targeting therapeutics: molecular mechanisms of antisense oligonucleotides as a therapeutic platform. Annu. Rev. Pharmacol. Toxicol. 50:259-93.

31. O'Brien S, et al. (2009) 5-year survival in patients with relapsed or refractory chronic lymphocytic leukemia in a randomized, phase III trial of fludarabine plus cyclophosphamide with or without oblimersen. J. Clin. Oncol. 27:5208-12.

32. Chi KN, et al. (2005) A phase I pharmacokinetic and pharmacodynamic study of OGX-011, a 2'-methoxyethyl antisense oligonucleotide to clusterin, in patients with localized prostate cancer. J. Natl. Cancer Inst. 97:1287-96.

33. Kastelein JJ, et al. (2006) Potent reduction of apolipoprotein B and low-density lipoprotein cholesterol by short-term administration of an antisense inhibitor of apolipoprotein B. Circulation. 114:1729-35.

34. Agarwala A, Jones P, Nambi V. (2015) The role of antisense oligonucleotide therapy in patients with familial hypercholesterolemia: risks, benefits, and management recommendations. Curr. Atheroscler. Rep. 17:467.

35. MacLeod AR. (2013) Antisense therapies for cancer: Bridging the pharmacogenomic divide. Drug Discov. Today Ther. Strateg. 10: e157-e163.

36. Juliano RL, Ming X, Nakagawa O. (2012) Cellular uptake and intracellular trafficking of antisense and siRNA oligonucleotides. Bioconjug. Chem. 23:147-57.

37. Castanotto D, Stein CA. (2014) Antisense oligonucleotides in cancer. Curr. Opin. Oncol. 26:584-9.

38. Moreno PM, Pego AP. (2014) Therapeutic antisense oligonucleotides against cancer: hurdling to the clinic. Front. Chem. 2:87.

39. Walker I, Irwin WJ, Akhtar S. (1995) Improved cellular delivery of antisense oligonucleotides using transferrin receptor antibodyoligonucleotide conjugates. Pharm. Res. 12:1548-53.

40. Uckun FM, Qazi S, Dibirdik I, Myers DE. (2013) Rational design of an immunoconjugate for selective knock-down of leukemia-specific E2APBX1 fusion gene expression in human Pre-B leukemia. Integr. Biol. 5:122-32.

41. Nitschke L. (2005) The role of CD22 and other inhibitory co-receptors in B-cell activation. Curr. Opin. Immunol. 17:290-7.

42. Tedder TF, Poe JC, Haas KM. (2005) CD22: a multifunctional receptor that regulates $\mathrm{B}$ lymphocyte survival and signal transduction. Adv. Immunol. 88:1-50.

43. Boue DR, LeBien TW. (1988) Expression and structure of CD22 in acute leukemia. Blood. 71:1480-6.

44. Grandori C, Cowley SM, James LP, Eisenman RN. (2000) The Myc/Max/Mad network and the transcriptional control of cell behavior. Annu. Rev. Cell Dev. Biol. 16:653-99.

45. Yun JS, Rust JM, Ishimaru T, Diaz E. (2007) A novel role of the Mad family member Mad3 in cerebellar granule neuron precursor proliferation. Mol. Cell. Biol. 27:8178-89.

46. Barisone GA, Yun JS, Diaz E. (2008) From cerebellar proliferation to tumorigenesis: new insights into the role of Mad3. Cell Cycle. 7:423-7.

47. Satake N, et al. (2014) Targeted therapy with MXD3 siRNA, anti-CD22 antibody and nanoparticles for precursor B-cell acute lymphoblastic leukaemia. Br. J. Haematol. 167:487-99.

48. Barisone GA, et al. (2014) Loss of MXD3 induces apoptosis of Reh human precursor B acute lymphoblastic leukemia cells. Blood Cells Mol. Dis. 54:329-35.

49. Seth PP, et al. (2009) Short antisense oligonucleotides with novel $2^{\prime}-4^{\prime}$ conformationaly restricted nucleoside analogues show improved potency without increased toxicity in animals. J. Med. Chem. 52:10-13.

50. Agard NJ, Prescher JA, Bertozzi CR. (2004) A strain-promoted [3 + 2] azide-alkyne cycloaddition for covalent modification of biomolecules in living systems. J. Am. Chem. Soc. 126:15046-7. 
51. Engel P, et al. (1993) The same epitope on CD22 of $B$ lymphocytes mediates the adhesion of erythrocytes, $\mathrm{T}$ and $\mathrm{B}$ lymphocytes, neutrophils, and monocytes. J. Immunol. 150:4719-32.

52. Burgess A, et al. (2010) Loss of human Greatwall results in G2 arrest and multiple mitotic defects due to deregulation of the cyclin B-Cdc2/ PP2A balance. Proc. Natl. Acad. Sci. U. S. A. 107:12564-9.

53. Pizzo PA, Poplack DG. Principles and Practice of Pediatric Oncology. Philadelphia: Lippincott Williams \& Wilkins. 1320 pp.

54. Kato J, et al. (2013) Efficacy of a CD22-targeted antibody-saporin conjugate in a xenograft model of precursor-B cell acute lymphoblastic leukemia. Leuk. Res. 37:83-8.

55. Ratheesh A, et al. (2012) Centralspindlin and alpha-catenin regulate Rho signalling at the epithelial zonula adherens. Nat. Cell Biol. 14:818-28.

56. Pathak D, Sepp KJ, Hollenbeck PJ. (2010) Evidence that myosin activity opposes microtubule-based axonal transport of mitochondria. J. Neurosci. 30:8984-92.

57. McManus MT, Sharp PA. (2002) Gene silencing in mammals by small interfering RNAs. Nat. Rev. Genet. 3:737-47.

58. Chan JH, Lim S, Wong WS. (2006) Antisense oligonucleotides: from design to therapeutic application. Clin. Exp. Pharmacol. Physiol. 33:533-40.

59. Evers MM, Toonen LJ, van Roon-Mom WM. (2015) Antisense oligonucleotides in therapy for neurodegenerative disorders. Adv. Drug Deliv. Rev. 87:90-103.

60. Kole R, Krainer AR, Altman S. (2012) RNA therapeutics: beyond RNA interference and antisense oligonucleotides. Nat. Rev. Drug Discov. 11:125-40.

61. Carnahan J, et al. (2007) Epratuzumab, a CD22-targeting recombinant humanized antibody with a different mode of action from rituximab. Mol. Immunol. 44:1331-41.

62. Raetz EA, et al. (2008) Chemoimmunotherapy reinduction with epratuzumab in children with acute lymphoblastic leukemia in marrow relapse: a Children's Oncology Group Pilot Study. J. Clin. Oncol. 26:3756-62.

63. Hochberg J, El-Mallawany NK, Cairo MS. (2014) Humoral and cellular Immunotherapy in ALL in Children, Adolescents, and Young Adults. Clin. Lymphoma Myeloma Leuk. 14s: S6-s13.

64. Mussai F, et al. (2010) Cytotoxicity of the anti-CD22 immunotoxin HA22 (CAT-8015) against paediatric acute lymphoblastic leukaemia. Br. J. Haematol. 150:352-8.

65. O'Donnell RT, Ma Y, McKnight HC, Pearson D, Tuscano JM. (2009) Dose, timing, schedule, and the choice of targeted epitope alter the efficacy of anti-CD22 immunotherapy in mice bearing human lymphoma xenografts. Cancer Immunol. Immunother. CII 58:2051-8.

66. Li D, et al. (2013) DCDT2980S, an anti-CD22monomethyl auristatin $\mathrm{E}$ antibody-drug conjugate, is a potential treatment for non-Hodgkin lymphoma. Mol. Cancer Ther. 12:1255-65.
67. Pfeifer M, et al. (2015) Anti-CD22 and anti-CD79B antibody drug conjugates are active in different molecular diffuse large B-cell lymphoma subtypes. Leukemia. 29:1578-86.

68. McLaughlin $P$, et al. (1998) Rituximab chimeric anti-CD20 monoclonal antibody therapy for relapsed indolent lymphoma: half of patients respond to a four-dose treatment program. J. Clin. Oncol. 16:2825-33.

Cite this article as: Satake N, et al. (2016) Novel targeted therapy for precursor B-cell acute lymphoblastic leukemia: Anti-CD22 antibody-MXD3 antisense oligonucleotide conjugate. Mol. Med. 22:632-42. 